\title{
Statistical Analysis of Pharmacokinetic Models in Dynamic Contrast-Enhanced Magnetic Resonance Imaging
}

\author{
Volker J. Schmid ${ }^{1}$, Brandon J. Whitcher ${ }^{2}$, Guang-Zhong Yang ${ }^{1}$, \\ N. Jane Taylor ${ }^{3}$, and Anwar R. Padhani ${ }^{3}$
}

1 Institute for Biomedical Engineering, Suite 5, Sherfield Building, Imperial College, South Kensington, London SW7 2AZ, United Kingdom

\{v.schmid, g.z.yang\}@imperial.ac.uk

2 Translational Medicine \& Genetics, GlaxoSmithKline, Greenford Road,

Greenford UB6 0HE, Middlesex, United Kingdom

brandon.j.whitcher@gsk.com

3 Paul Strickland Scanner Centre, Mount Vernon Hospital, Rickmansworth Road,

Northwood HA6 2RN, Middlesex, United Kingdom

\begin{abstract}
This paper assesses the estimation of kinetic parameters from dynamic contrast-enhanced magnetic resonance imaging (DCEMRI). Asymptotic results from likelihood-based nonlinear regression are compared with results derived from the posterior distribution using Bayesian estimation, along with the output from an established software package (MRIW). By using the estimated error from kinetic parameters, it is possible to produce more accurate clinical statistics, such as tumor size, for patients with breast tumors. Further analysis has also shown that Bayesian methods are more accurate and do not suffer from convergence problems, but at a higher computational cost.
\end{abstract}

\section{Introduction}

The quantitative analysis of dynamic contrast-enhanced magnetic resonance imaging (DCE-MRI) is typically achieved by applying pharmacokinetic (PK) models to the signal intensity, or a nonlinear transformation of it, observed from the scanning process. The contrast agent Gd-DTPA (gadolinium diethylenetriaminepentaacetic acid) is a small molecular weight substance injected after several baseline scans have been acquired. Using $T_{1}$-weighted sequences, the reduction in $T_{1}$ relaxation time caused by the contrast agent is the dominant enhancement observed [1]. $T_{1}$-weighted kinetic curves typically have three phases: the upslope, maximum enhancement, and washout [2]. Quantitative PK parameters are estimated by fitting a nonlinear function, the solution of a system of linear differential equations, to the observations. Each PK parameter has a direct relationship with key biological processes of interest; e.g., volume transfer, leakage space, etc. This is a distinct advantage over the semi-quantitative approach where descriptive statistics of the kinetic curve (onset time, mean rate 
of change, maximum signal intensity, etc.) are estimated, but lack direct tissue or vascular information.

From a statistical point of view this quantitative methods are based on the theory of nonlinear regression 3. Non-linear models are typically hard to estimate due to optimization problems, but standard software is available. We estimate parameters in pharmacokinetic (PK) models using nonlinear regression in both a likelihood and a Bayesian framework 4 to help alleviate some of the concerns stated above and provide a richer summary of the results, specifically with respect to convergence issues.

Potential clinical applications of DCE-MRI include screening for malignant disease, lesion characterization, monitoring lesion response to treatment, and assessment of residual disease. Newer applications include prognostication, pharmacodynamic assessments of antivascular anticancer drugs, and predicting efficacy of treatment. We propose to look at parameters obtained from the two methods and how they impact clinically relevant statistics such as tumor size.

\section{Theory and Methods}

\section{$2.1 \quad$ DCE-MRI Data}

To evaluate our methods we use a dataset provided by the Paul Strickland Scanner Centre at the Mount Vernon Hospital, Northwood. The data consist of six patients with breast tumors, scanned once at the beginning of treatment and again after six weeks. The scans were acquired with a $1.5 \mathrm{~T}$ Siemens MAGNETOM Symphony scanner, $\mathrm{TR}=11 \mathrm{~ms}$ and $\mathrm{TE}=4.7 \mathrm{~ms}$. Each scan consists of three slices of $230 \times 256$ voxels. A dose of $D=0.1 \mathrm{mmol} / \mathrm{kg}$ body weight $\mathrm{Gd}-$ DTPA was injected at the start of the fifth acquisition using a power injector.

We use a standard compartmental model [5] to describe the arterial influx of Gd-DTPA into extracellular extravascular space (EES) and its venous efflux. The time series of gadolinium concentration in the tissue is modeled by

$$
C_{t}(t)=K^{\text {trans }}\left[C_{p}(t) \otimes \exp \left(-k_{\mathrm{ep}} t\right)\right]
$$

where $C_{t}(t)$ is the observed Gd-DTPA concentration in the tissue at time $t$ and $C_{p}(t)$ is the tracer concentration in arterial blood. The parameter $K^{\text {trans }}$ represents the transfer from plasma to EES, and $k_{\text {ep }}$ is the rate parameter for transport from the EES to plasma. Here, $\otimes$ denotes the convolution operator. The volume of EES per unit volume of tissue (leakage space) is given by

$$
v_{e}=\frac{K^{\mathrm{trans}}}{k_{\mathrm{ep}}} .
$$

For the arterial input function $C_{p}(t)$ we follow the work of Tofts and Kermode [6] and use a bi-exponential function

$$
C_{p}(t)=D \sum_{i=1}^{2} a_{i} \exp \left(-m_{i} t\right)
$$


with the values $a_{1}=3.99 \mathrm{~kg} / \mathrm{l}, a_{2}=4.78 \mathrm{~kg} / 1, m_{1}=0.144 \mathrm{~min}^{-1}$ and $m_{2}=$ $0.0111 \mathrm{~min}^{-1}$ and the actual dose per body weight.

To calculate the Gadolinium concentration $C_{t}$, the signal intensity was converted to $T_{1}$ relaxation time values using $T_{1}$-weighted images, proton density weighted images and data from a calibration experiment consisting of phantoms with known $T_{1}$ relaxation time. The Gd-DTPA concentration can then be computed via

$$
C_{t}(t)=\frac{1}{r_{1}}\left[\frac{1}{T_{1}(t)}-\frac{1}{T_{10}}\right]
$$

where $T_{10}$ is the $T_{1}$ value without contrast, computed as mean value of the first four images, and $r_{1}=4.24 \mathrm{l} / \mathrm{s} / \mathrm{mmol}$ is the longitudinal relativity of protons in vivo due to Gd-DTPA.

Regions of interest (ROIs) were drawn manually, on a scan-by-scan basis, using subtraction images from the acquisition of dynamic data. Although the tumor was isolated, in order to save on computation time, enough surrounding tissue was also captured to allow for reasonable contrast between tissue types within the ROI.

\subsection{Statistical Models}

Likelihood Approach. In each voxel we fitted a nonlinear regression model to the Gd-DTPA concentration time series. By carrying out the convolution in Eqn. 1, the following statistical model can be derived:

$$
C_{t}(t)=D \exp \left(\theta_{1}\right) \sum_{i=1}^{2} \frac{a_{i}\left\{\exp \left(-m_{i} t\right)-\exp \left[-\exp \left(\theta_{2}\right) t\right]\right\}}{\exp \left(\theta_{2}\right)-m_{i}}+\epsilon_{t}
$$

where $\epsilon_{t}$ is the noise error at time $t$. We assume the expected value of the error to be zero; i.e. $E(\epsilon)=0$. Inference is performed by minimizing the sum of squares of the errors $\min \sum \epsilon_{t}^{2}$. We use parameters $\exp \left(\theta_{1}\right)$ instead of $K^{\text {trans }}$ and $\exp \left(\theta_{2}\right)$ instead of $k_{\text {ep }}$ to insure positive values of $K^{\text {trans }}$ and $k_{\text {ep }}$. The parameter $v_{e}$ was computed using Eqn. 2, Estimation of the model was done using the nls function of the statistical computing environment $R$ [7]. The function uses a Gauss-Newton algorithm to estimate parameters of interest along with their standard errors.

As comparting models are typically difficult to optimize, we use a multiple search start point approach for the algorithm 8. To identify the starting values, we follow the ideas of Pinheiro and Bates [9] for first-order compartmental models. Nevertheless, the algorithm still failed to converge in $5 \%$ of voxels.

Bayesian Approach. For the Bayesian approach we use the following model

$$
C_{t}(t)=D K^{\mathrm{trans}} \sum_{i=1}^{2} \frac{a_{i}\left[\exp \left(-m_{i} t\right)-\exp \left(-k_{\mathrm{ep}} t\right)\right]}{k_{\mathrm{ep}}-m_{i}}+\epsilon .
$$


In the Bayesian framework prior probability density functions (PDFs) have to be specified for all unknown parameters, and hence, we use

$$
\begin{aligned}
\epsilon & \sim \mathrm{N}\left(0, \tau_{\epsilon}^{-1}\right), \\
\log \left(K^{\text {trans }}\right) & \sim \mathrm{N}\left(0, \tau_{K^{\text {trans }}}^{-1}\right), \\
\log \left(k_{\mathrm{ep}}\right) & \sim \mathrm{N}\left(0, \tau_{k_{\mathrm{ep}}}^{-1}\right),
\end{aligned}
$$

where $\mathrm{N}$ denotes the Gaussian (Normal) distribution. The variance parameters for these PDFs are also specified by so-called hyper-priors,

$$
\begin{aligned}
\tau_{\epsilon} & \sim \mathrm{Ga}(0.001,0.001), \\
\tau_{K^{\text {trans }}} & \sim \mathrm{Ga}(0.001,0.001), \\
\tau_{k_{\mathrm{ep}}} & \sim \mathrm{Ga}(0.001,0.001),
\end{aligned}
$$

where Ga denotes the Gamma distribution. So the PDFs of $\epsilon, K^{\text {trans }}$ and $k_{\mathrm{ep}}$ have high variance and therefore are very diffuse. This reflects lack of prior information about the parameters of interest. By using a normal prior distribution for $\log \left(K^{\text {trans }}\right)$ and $\log \left(k_{\text {ep }}\right)$, we ensure positive values for both parameters as with the likelihood method.

Inference is made by computing the posterior distribution $p\left(K^{\text {trans }}, k_{\mathrm{ep}} \mid C_{t}\right)$ given by Bayes' theorem, so that

$$
p\left(K^{\text {trans }}, k_{\mathrm{ep}} \mid C_{t}\right)=\frac{p\left(K^{\text {trans }}, k_{\mathrm{ep}}\right) \ell\left(C_{t} \mid K^{\text {trans }}, k_{\mathrm{ep}}\right)}{\int p\left(K^{\text {trans }}, k_{\mathrm{ep}^{*}}\right) \ell\left(C_{t} \mid K^{\text {trans }}, k_{\mathrm{ep}^{*}}\right)},
$$

where $\ell\left(C_{t} \mid K^{\text {trans }}, k_{\mathrm{ep}}\right)$ denotes the likelihood function of $C_{t}(t)$. Samples from the posterior distribution are obtained via Markov chain Monte Carlo (MCMC) methods 4] implemented in $\mathrm{C}++$. For estimating the parameter and standard errors we use the mean and the empirical standard error of the sample of each parameter, respectively, after a reasonable burn-in phase.

MRIW. Estimation of pharmacokinetic parameters was also performed using MRIW v4.1.6 10], an established software package for parametric analysis of DCE-MRI data developed at the Institute of Cancer Research, United Kingdom.

\section{Results}

Both the likelihood and Bayesian methods were applied to six patients, with breast tumor, before and after treatment. Fig. 11 shows the estimated volume transfer constant $\left(K^{\text {trans }}\right)$, the standard error of $\log \left(K^{\text {trans }}\right)$ and the leakage space $\left(v_{e}\right)$ from a single slice of patient \#2 before therapy. The tumors are clearly visible as a region of high $K^{\text {trans }}$, although the likelihood-based procedure and MRIW failed to converge for approximately $5 \%$ of voxels both inside and outside the tumor. Bayesian parameter estimates were available for all voxels. When looking at leakage space the tumor is surrounded by a region of high $v_{e}$, making the edges of the tumor difficult to identify. 
$K^{\text {trans }}$

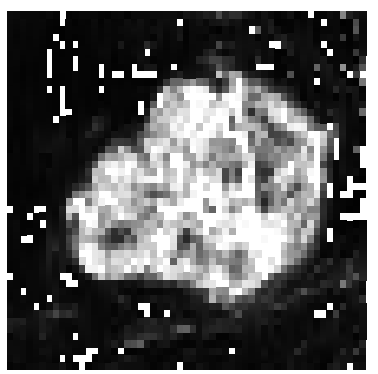

(2)

(3)
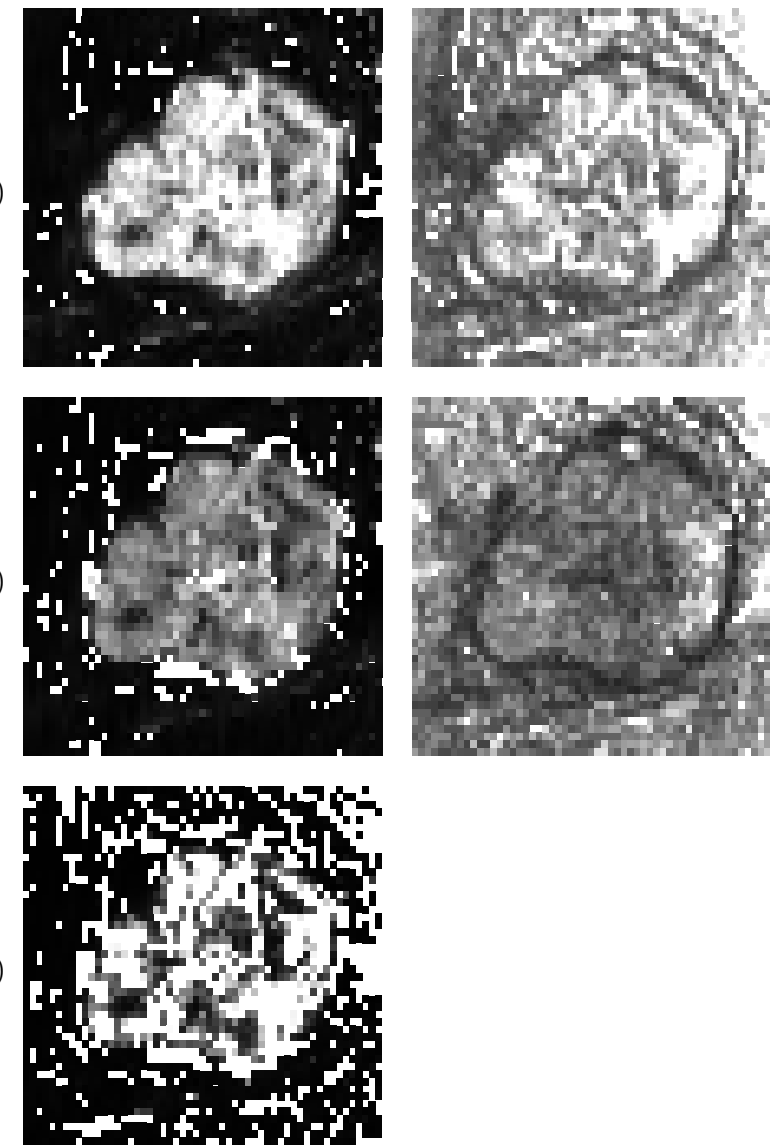

$v_{e}$
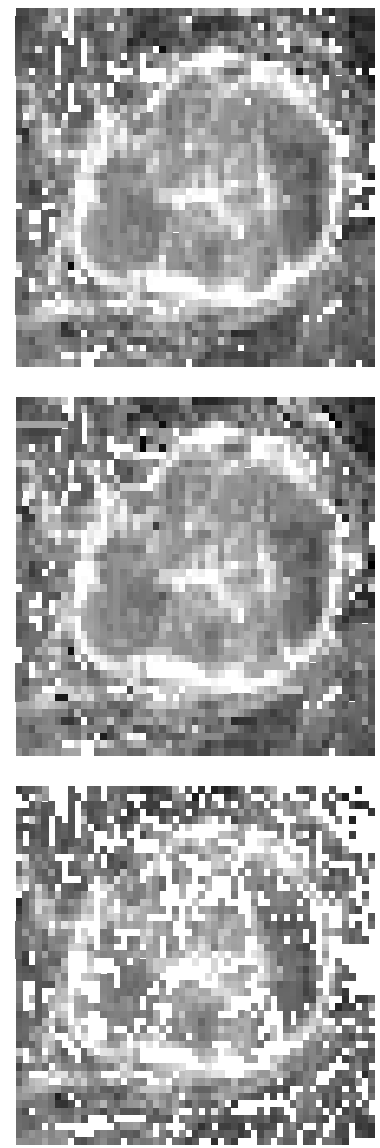

Fig. 1. Parametric maps of kinetic parameters for a region of interest around a breast tumor - from left to right - the volume transfer constant $\left(K^{\text {trans }}\right)$, the standard error of $\log \left(K^{\text {trans }}\right)$ and the leakage space $\left(v_{e}\right)$. The rows correspond to three methods likelihood (1), Bayesian (2) and MRIW (3).

To specify the exact region of the tumor, we threshold $K^{\text {trans }}$ such that only values exceeding 0.3 remain (Fig. 2, left). This helps to highlight the tumor, but many voxels not associated with the tumor also remain. To produce a better specification, we take advantage of the estimated standard error for $K^{\text {trans }}$. Fig. 1 (right) shows the estimated standard error of $\log \left(K^{\text {trans }}\right)$ for the likelihood and Bayesian methods (see our model specification in Sec.2.2). The error is especially high where the estimated value of $K^{\text {trans }}$ is high (e.g., in the tumor). Assuming an asymptotic Normal distribution, we can compute the probability of each pixel exceeding the threshold. Fig. 2 (middle) shows this probability map, whereas Fig. 2 (right) shows pixels where the probability of exceeding the threshold 
(i.e., a pixel being part of the tumor) is greater than or equal to $99 \%$. Utilizing the PDF of $K^{\text {trans }}$, either its asymptotic distribution via the likelihood method or its posterior distribution via the Bayesian method, produces a much better separation between tumor and non-tumor voxels.

We explore the clinical application of these methods by computing the size of the tumor, defined here to be the number of voxels per slice. A tumor mask was created from the estimated $K^{\text {trans }}$ for each method (Fig. 3) and the size of the tumor, both pre- and post-treatment, is provided in Tab. 1 for all six patients. There is good agreement between the three methods for most of the scans.

(1)

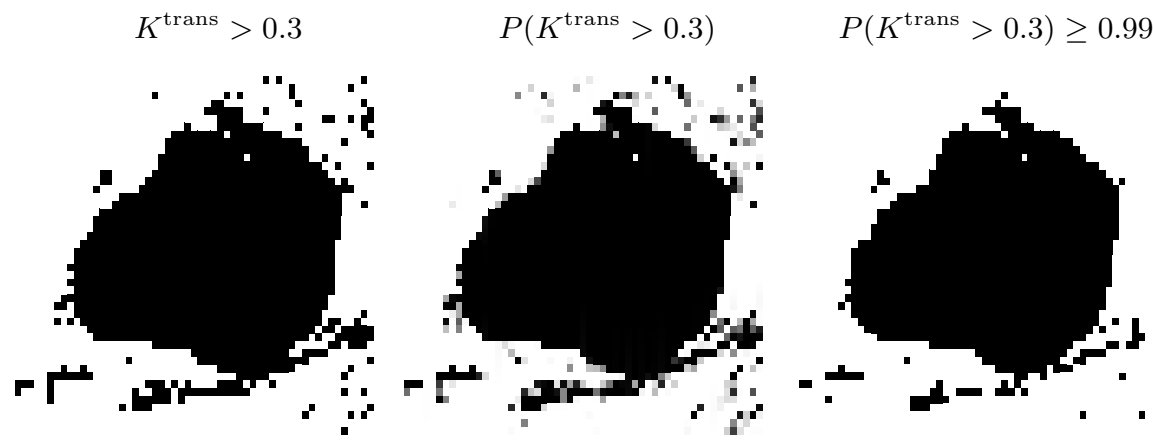

(2)
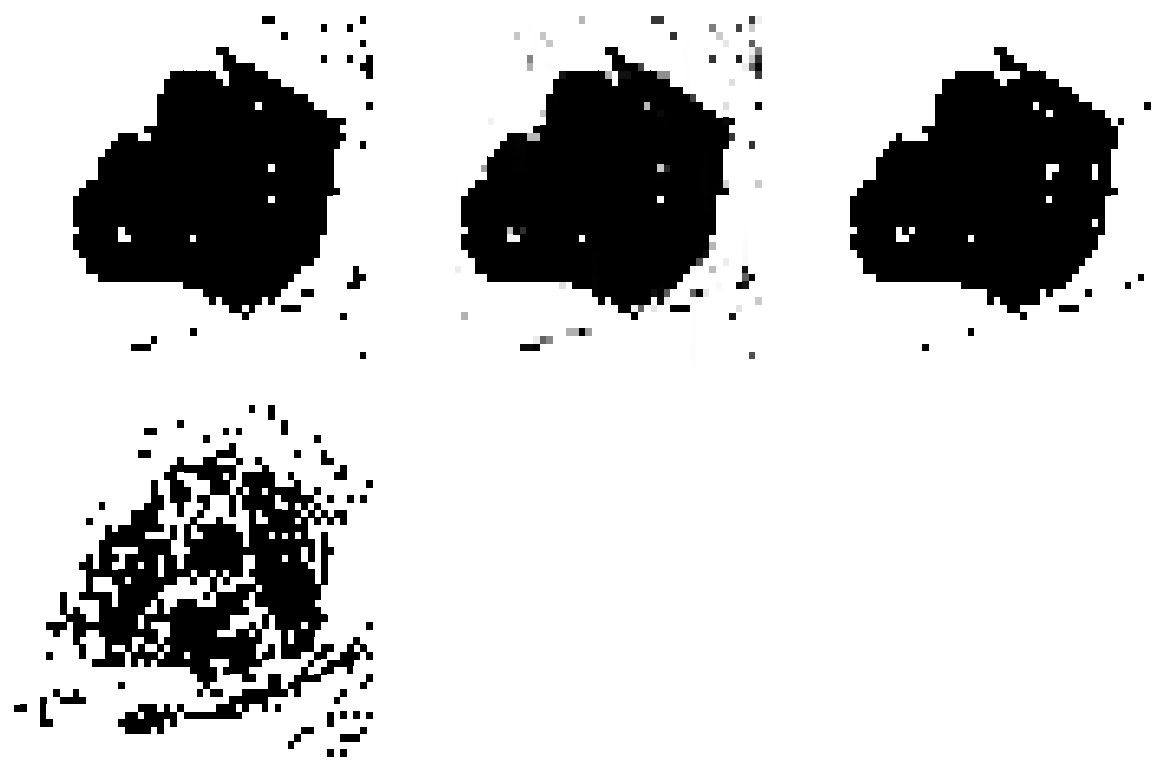

$(3)$

Fig. 2. Threshold maps derived from estimates of $K^{\text {trans }}$ of first scan of patient \#2 from left to right - basic thresholding, probability of exceeding the threshold and probability of exceeding the threshold by at least $99 \%$. The rows correspond to three methods - from top to bottom - likelihood (1), Bayesian (2) and MRIW (3). 
Likelihood

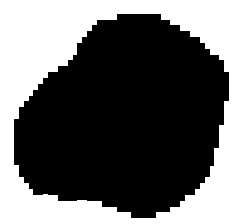

Bayesian

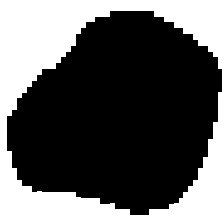

MRIW

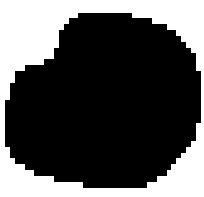

Fig. 3. Mask of the tumor of scan 1 of patient \# 2 based on different estimates of $K^{\text {trans }}-$ from left to right - likelihood, Bayesian and MRIW

Table 1. Tumor size (number of voxels) derived from $K^{\text {trans }}$ for each slice of the six patients, pre- and post-treatment

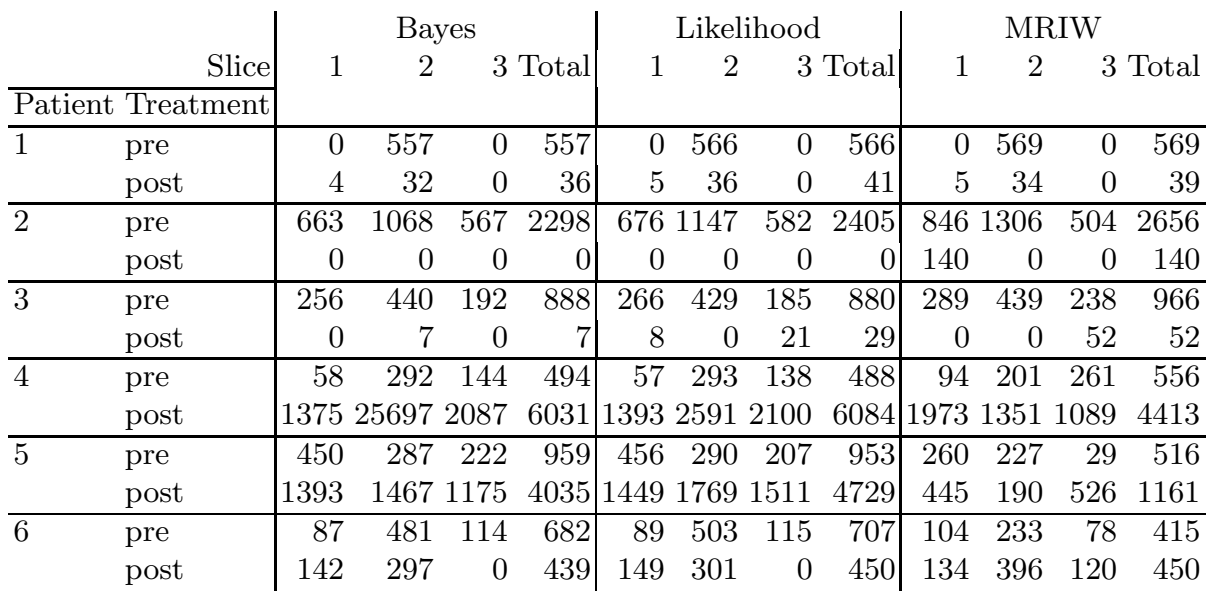

\section{Conclusion}

Statistical properties of pharmacokinetic parameters from DCE-MRI have been used to improve clinically relevant quantities of interest. Bayesian techniques allow one to input prior information into the estimation procedure, greatly reducing problems with convergence with the cost of increased computing time. The posterior distribution provides instant access to valuable information about the kinetic parameters without resorting to asymptotic results. We have utilized second-order quantities of the posterior distribution to help discriminate voxels and produce more accurate summaries of clinically meaningful statistics.

With the proposed framework, it is possible to incorporate additional information via the prior distributions including dependence between kinetic parameters and spatial constraints, thus moving towards semi-parametric or nonparametric models in DCE-MRI. 


\section{Acknowledgments}

Support for Dr. Schmid was financed through a research grant from GlaxoSmithKline. The clinical data was graciously provided by Dr. A.R. Padhani.

\section{References}

1. Parker, G.J.M., Padhani, A.R.: $T_{1}$-w DCE-MRI: $T_{1}$-weighted dynamic contrastenhanced MRI. In Tofts, P., ed.: Quantitative MRI of the Brain. Wiley, Chichester, England (2003) 341-364

2. Collins, D.J., Padhani, A.R.: Dynamic magnetic resonance imaging of tumor perfusion. IEEE Engineering in Biology and Medicine Magazine (2004) 65-83

3. Seber, G.A.F., Wild, C.J.: Nonlinear Regression. Wiley, Hoboken, New Jersey (1988)

4. Gilks, W.R., Richardson, S., Spiegelhalter, D.J.: Markov Chain Monte Carlo in Practice. Chapman \& Hall, London (1996)

5. Kety, S.: Blood-tissue exchange methods. Theory of blood-tissue exchange and its applications to measurement of blood flow. Methods in Medical Research 8 (1960) 223-227

6. Tofts, P., Kermode, A.: Measurement of the blood-brain barrier permeability and leakage space using dynamic MR imaging-1. Fundamental concepts. Magn Reson Med 17 (1991) 357-36

7. R Development Core Team: R: A language and environment for statistical computing. R Foundation for Statistical Computing, Vienna, Austria. (2003) ISBN 3-900051-00-3.

8. Ahearn, T., Staff, R., Redpath, T., Semple, S.: The use of the Levenberg-Marquardt curve-fitting algorithm in pharmacokinetic modelling of DCE-MRI. Physics in Medicine and Biology 50 (2005) N85-N92

9. Pinheiro, J.C., Bates, D.M.: Mixed-Effects Models in S and S-PLUS. SpringerVerlag, New York (2000)

10. Parker, G., Suckling, J., Tanner, S., Padhani, A., Husband, J., Leach, M.: MRIW: parametric analysis software for contrast-enhanced dynamic mr imaging in cancer. Radiographics 18 (1998) 497-506 